\title{
Gender, Age and Emotion Recognition using Deep Learning
}

\author{
Siddharth G S, Abhinav Ram Bhatta, Sanath Kumar G V, Sarveshvar M R \\ Jain University - School of Engineering and Technology \\ Bangalore, Karnataka, India
}

\begin{abstract}
Automatic gender and age recognition have now pertinent to an extension of its usage in various software and hardware, particularly because of the growth of online social networking websites and social media. The advertisements can be specialized based on the age and the gender of the person on the phone. It also can help identify suspects in criminal cases or at least it can minimize the number of suspects. A Convolution Neural Network is a deep neural network (DNN) widely used for the purposes of image recognition and processing and NLP. A convolution neural network architecture is built and the model is trained for gender, age and emotion recognition from images. An android application can be developed implementing the age, gender and emotion recognition. With the phone capturing the photo, the frames are pre-processed and fed to the model to accomplish this task, and the prediction is displayed accordingly.
\end{abstract}




\section{Introduction}

This is an overview of gender, age and emotion recognition is provided. The importance of gender, age and emotion recognition and the role of IT in gender, age and emotion recognition is also provided.

\section{Overview of Gender, Age and Emotion Recognition}

A change of paradigm is currently underway. Computers have been consistently surpassing humans in many tasks of the day-to-day life in the past decades, with us relying more and more on them. If a bank's database servers would for some reason shutdown and wipe out all of its information, the world economy could collapse. We are in the age of information, and that information is increasingly being collected and stored by machines. Machine Learning uses this information to learn patterns. It can be used to predict the weather, to help on credit approval, or to recommend a visit to the doctor if an irregular heartbeat is detected.

But this change of paradigm is currently occurring in a specific subfield of computer science - computer vision, fueled by the advent of Deep Learning and Convolutional Neural Networks. Over the last decade, the rate of image uploads to the internet has been increasing exponentially. Motivated by this and the fact that high computing power is becoming increasingly accessible to the common user, more and more research is being done on computer vision. Problems that were once considered impossible to study due to lack of data or computing power are being attempted now. Cars can drive themselves nowadays, detecting pedestrians and predicting/avoiding accidents. Despite self-driving cars being a hot-topic today, there is perhaps one even hotter - facial analysis.

Facial analysis comprehends several tasks, which can be used for several purposes. There are models for the task of face detection, pose estimation, age estimation, face recognition, smile detection, gender recognition, and so on. In many cases, machine-estimation has been consistently breaking the human level, achieving outstanding results. There is a sense of urgency in the quest to investigate and develop the best model, fueled by Artificial Intelligence competitions and the tech business world. These systems can be applied to security systems, person identification, human-computer interaction platforms, marketing, etc. We are getting closer, step by step, to become fully connected to technology, which can now lea and estimate our needs and characteristics. Facial analysis plays a key role in this scenario, as a significant part of human communication is nonverbal.

\section{Importance of gender, age and emotion recognition}

Facial analysis systems can ultimately change the way we live. Allied to Artificial Intelligence, it can provide personal assistance in every aspect of life. It can estimate our mood upon waking and, connecting it to our habits, age and other factors, provide the perfect meal for the moment, the best music to accompany that meal, and the perfect outfit, linked to the weather prediction. Likewise, upon entering a clothing store, it can automatically suggest the best piece of clothing for you, according to your age and gender, and send to your smartphone a picture of you virtually dressed in such suggestions. Machines will be capable of taking care of the uninteresting part of life, endto-end. Supported by computer vision and facial analysis, the full connection and understanding of humans will soon happen. In fact, many state-of-the-art models have already outpaced human recognition in many facial analysis tasks.

\section{Role of IT in gender, age and emotion recognition}

The state-of-the-art methods in image-related tasks such as image classification andobject detection are all based on Convolutional Neural Networks (CNNs). Human face analysis and soft-biometric classification, has gained more popularity after Alex Net has been introduced by Krizhevsky et al. Such facial soft-biometric include age, gender and facial expression are a topic of interest among many computer vision researchers. Introduction of deep learning to this domain has replaced the need for handcrafted facial attributes and data pre-processing schemes. D-CNN models have been not only successfully applied to Computer Vision.

\section{LITERATURE SURVEY}

The proposed design falls in the CNN framework. A Convolution Neural Network is a deep neural network (DNN) widely used for the purposes of image recognition and processing and NLP. 


\section{Deep Convolutional Neural Network for Age} Estimation based on VGG-Face Model

In this paper, a deep CNN model was trained on a database for face recognition task and was used to estimate the age information on the Adience database.

\section{Contributions:}

This paper has three significant contributions in this field.

1. This work proves that a CNN model, which was trained for face recognition task, can be utilized for age estimation to improve performance.

2. Over fitting problem can be overcome by employing a pretrained $\mathrm{CNN}$ on a large database for face recognition task.

3. Not only the number of training images and the number subjects in a training database effect the performance of the age estimation model, but also the pre-training task of the employed CNN determines the model's performance.

\section{Methodology:}

One of the most challenging problems in the machine learning is the overfitting problem that occurs when using small databases. In deep neural networks, the problem of the overfitting becomes even worse due to the fact that deep networks have millions of parameters, since they have several numbers of layers with thousands of nodes. All databases that are built for age classification and prediction are relatively small in size. They are not comparable in size with other databases designed for face recognition and image classification tasks. In the proposed deep CNN for age estimation by using a deep CNN model trained for face recognition on a very large database, to overcome the overfitting problem. The input images are rescaled to $256 \times 256$ pixel, then randomly cropped to $224 \times 224$ pixel patches. The optimization the network is carried out by using stochastic gradient descent method. The stochastic gradient decent method optimizes and finds the parameters of the connected layers that minimize the prediction of the SoftMax-log-loss for age estimation as shown below.

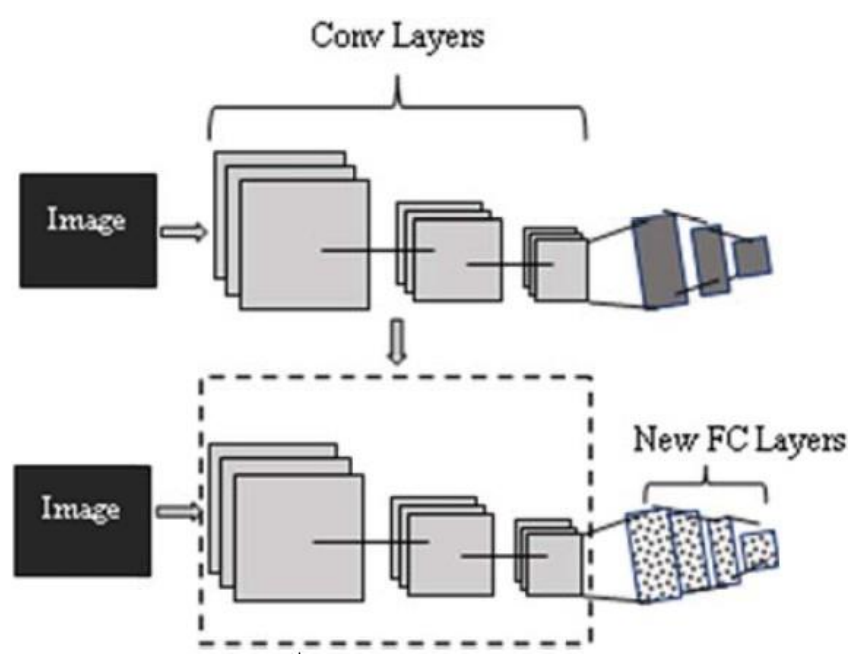

Advantages:

In the proposed deep $\mathrm{CNN}$ for age estimation the overfitting problem is solved by using a deep CNN model trained for face recognition on a very large database.

\section{Limitations:}

Not only the number of the training the number subjects in a training database effect the performance for age estimation, but also the pre-images and training task of the employed CNN determines the network's performance for age estimation.

\section{DAGER: Deep Age, Gender and Emotion Recognition} Using Convolutional Neural Networks

An end-to-end system capable of estimating facial attributes including age, gender and emotion with low error rates is discussed in this paper.

\section{Contributions:}

The contributions of this work are summarized as:

4. An end-to-end pipeline, along with novel deep networks, that not only are computationally inexpensive, but also outperform competitive methods on several benchmarks.

5. Large datasets for age, emotion and gender recognition are used to train state-of-the- art deep neural networks.

6. A number of experiments were conducted on existing benchmarks and obtained leading results on all of them. 


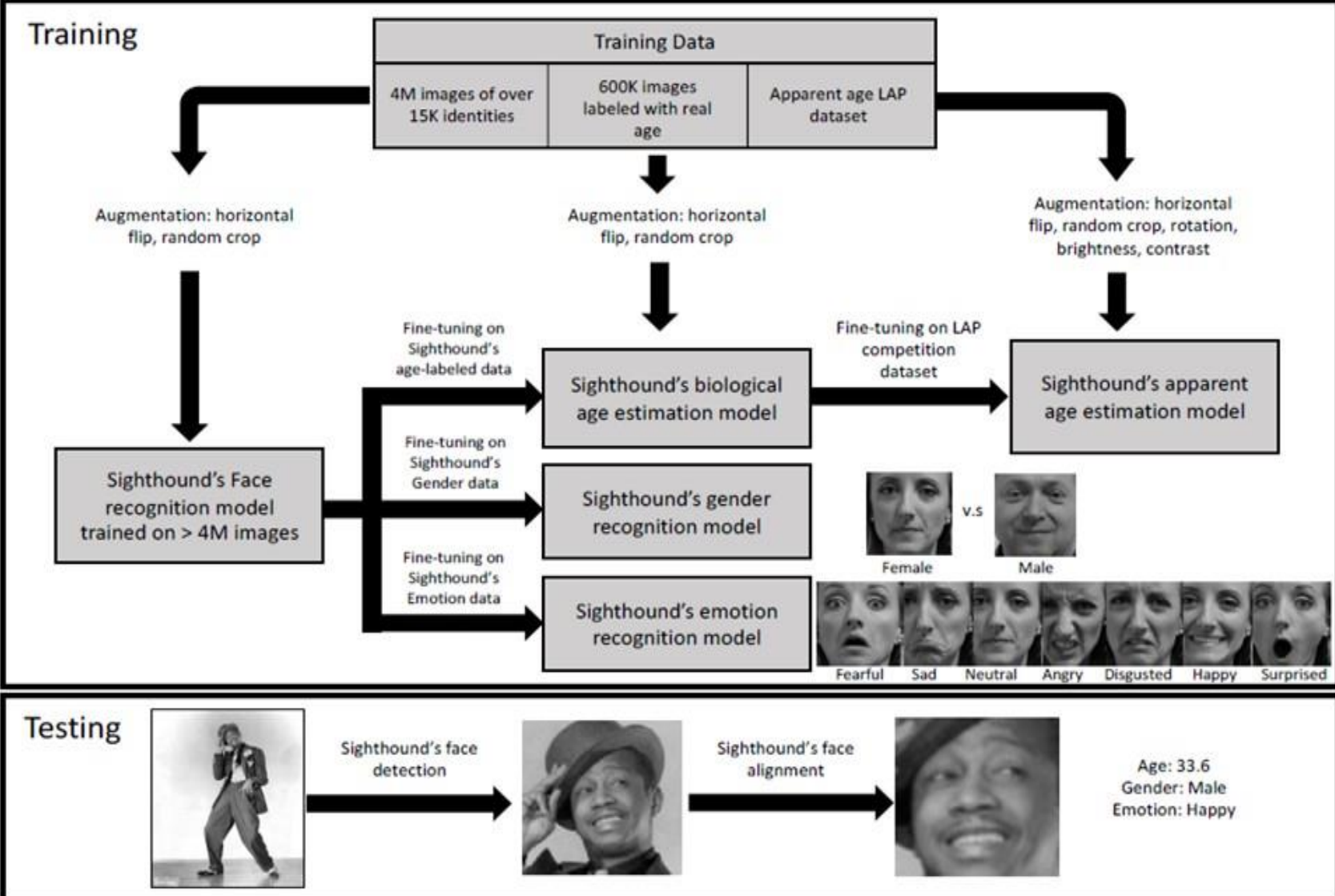

Methodology:

The first deep model is trained on a large dataset of four million images for the task of face recognition. This model serves as the backbone to facial attribute recognizers and is used to fine-tune networks for four tasks: real age estimation, apparent age estimation, gender recognition and emotion recognition. The figure shows the pipeline of the system. Images are collected from different sources and labeled for different tasks. Over 4 million images of more than 40,000 people are collected for the task of facial recognition. All images are labeled with their corresponding gender label and part of the data is annotated with emotion. These images are later pruned using a semiautomated process with a team of human annotators in the loop. The images are pre-processed next to extract the faces and align them. The aligned images are then fed to their proprietary deep network for training.

\section{Advantages:}

The novel deep architecture used along with the large in-house collected data, can outperform competitive commercial and academic algorithms on several benchmarks. Due to the release of large labeled datasets, as well as the advances made in the design of convolutional neural networks, error rates have dropped significantly.

\section{Limitations:}

Collecting labeled data for some tasks, such as real age estimation, is much more challenging compared to popular classification or detection problems. This disparity is due to the fact that human error in estimating real age is large (sometimes greater than the computer vision estimations) and one cannot rely on human annotators to label faces with their corresponding real age.

\section{Real-time Convolutional Neural Networks for Emotion and Gender Classification}

An implementation of general convolutional neural network building framework for designing real-time CNNs is provided in this paper.

\section{Contributions:}

Validation of the model by creating a real-time vision system which accomplishes the tasks of face detection, gender classification and emotion classification simultaneously in one blended step using our proposed

\begin{tabular}{llll}
\hline \hline (C) 2022, IJSREM & www.ijsrem.com & DOI: & Page 4
\end{tabular} 
CNN architecture. The success of service models decisively depends on a smooth model to user interaction. Thus, a model should be able to extract information just from the face of its user. Realtime visualization of the guided-gradient back-propagation is implemented in order to validate the features learned by the $\mathrm{CNN}$, establish a balance between their classification accuracy and unnecessary parameters.

\section{Methodology:}

The proposed technique has two models which are evaluated in accordance to the test accuracy and number of parameters. Both models were designed with the idea of creating the best accuracy over number of parameters ratio. First model relies on the idea of eliminating completely the fully connected layers. The second architecture combines the deletion of the fully connected layer and the inclusion of the combined depth-wise separable convolutions and residual modules. Diagram shows proposed model for realtime classification.

Fully connected layers tend to contain most of the parameters in a CNN. Specifically, VGG16 contains approximately $90 \%$ of all its parameters in their last fully connected layers. Recent architectures such as Inception V3, reduced the number of parameters in their last layers by including a Global Average Pooling operation. Global Average Pooling reduces each feature map into a scalar value by taking the average over all elements in the feature map. The average operation forces the network to extract global features from the input image.

\section{Advantages:}

The report accuracy of gender dataset is estimated to be $96 \%$ in the IMDB. The performance is faster here as small CNNs are used. A number of parameters used are less in order to alleviate from better slow performances and better generalization.

\section{Limitations:}

The drawbacks of this system are:

- The use of glasses might affect the emotion classification by interfering with the features learned.

- Misclassifications occurs since the training dataset consist of mostly western: actors, writers and cinematographers.
- The report accuracy of emotion dataset is estimated to be $66 \%$ in theFER-2013

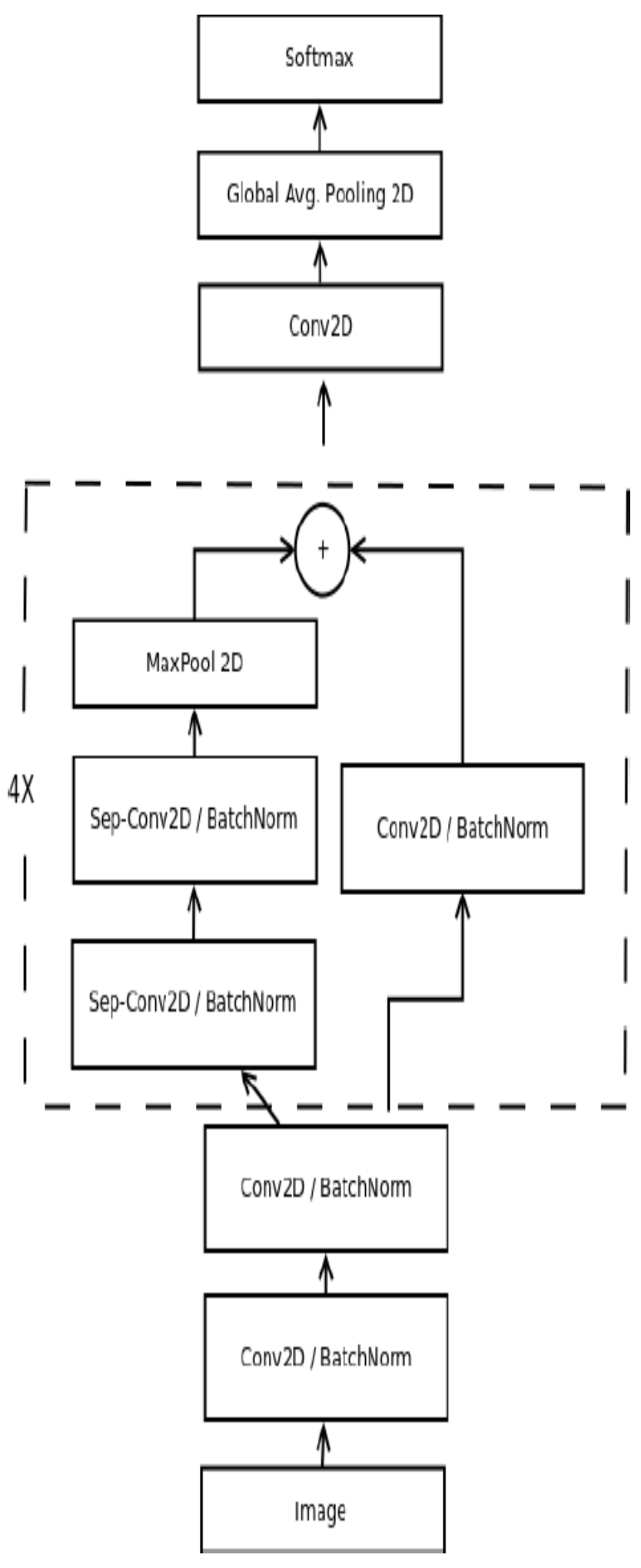




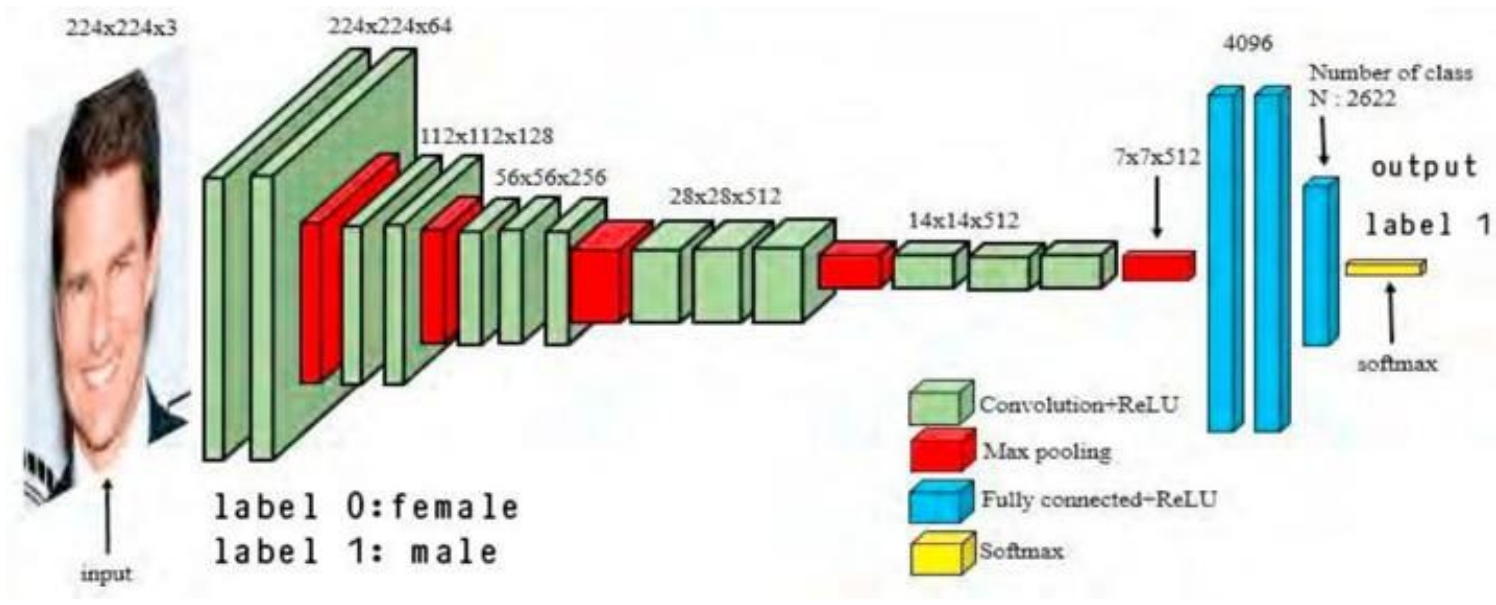

\section{Gender Recognition Through Face Using Deep Learning}

An implementation of domain-specific Deep Convolution neural network (D-CNN) method instead of generic Deep Convolution neural network(D-CNN) method is provided in this paper.

\section{Contributions:}

They have tried to show the determination of gender recognition through face image that can improve overall accuracy with VGGNet architecture of Deep Convolution Neural Network (D-CNN).

\section{Methodology:}

In this paper, VGGNet architecture of Deep Convolution Neural Network (D-CNN) for gender recognition, in order to improvise the previously used method and to obtain much accurate result. Figure depicts how to process face image through D-CNN and find the pattern, extract feature to recognize gender from image accurately. Already trained VGG-Face Deep Convolution Neural Network (CNN) method are fine tuned for the task of recognizing the gender of a human being from his/her face image. Figure shows age and gender recognition architecture. The VGGNet-face network has been trained to recognize more than 10000 celebrity IDs. With this human-computer intercommunication, supervision, vigilance device, and digital vision system and much more that will work on whole human presence. Nevertheless, in a physical world scenario, the challenge is how to do work with the face image which influences various factors like illumination, pose facial expression, age estimation, occlusion, and background instruction data, and noise, error.

\section{Advantages:}

Using D-CNN the features can be automatically extracted from an image and give output, no need to use feature descriptors like Histogram Oriented Gradient (HOG) and Support vector machine (SVM), eigenvector to extract the feature from image manually so as to do further recognition task or classification task.

\section{Limitations:}

The model is limited to learning only the six-basic emotion plus neutral. It conflicts with what is present in everyday life, which has emotions that are more complex.

\section{Age and Gender Prediction from Face Images Using Convolutional Neural Network}

This paper proposes an age and gender prediction method from face images using convolutional neural network.

\section{Contributions:}

This paper proposes an age and gender prediction method from face images using convolutional neural network. Through a set of experiments using public face databases, we demonstrate that the proposed method exhibits the efficient performance on age and gender prediction compared with conventional methods. This paper focuses on age and gender prediction from face images for the purpose of enhancing the performance of 
face recognition systems.

\section{Methodology:}

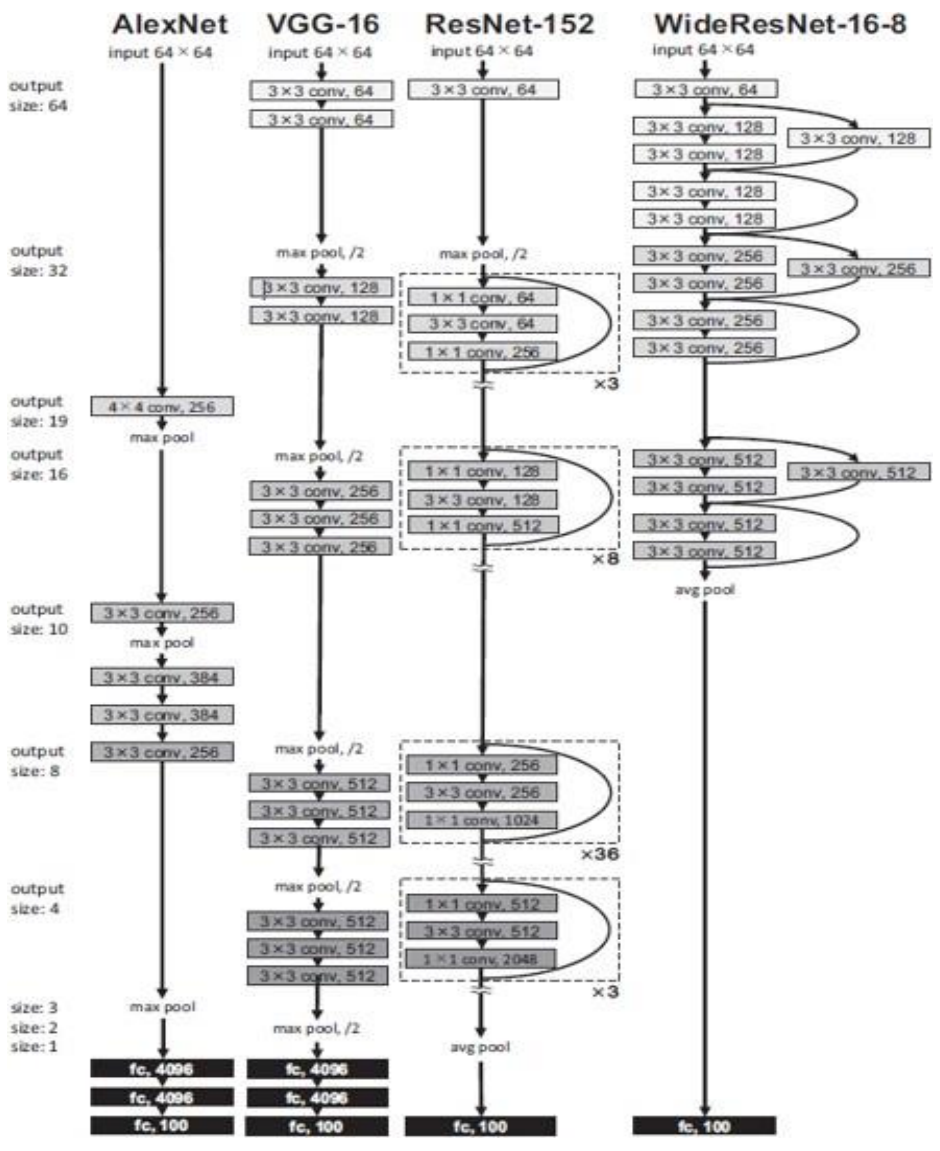

The general flow of face recognition consists of three steps: capture face images by a camera with visible or nearinfrared illumination, extract features from face images and evaluate the similarity between features. Figure 3.6 shows the different network architectures of $\mathrm{CNN}$.

A. Network Architecture:

The performance of $\mathrm{CNN}$ is directly influenced by the depth of network. Therefore, the recent network architectures such as GoogLeNet and WideResNet employ parallel network layers to exhibit the good performance of CNN. The large size of network exhibits the good performance on image recognition, while a huge amount of processing time or high-performance computers is required in training. Therefore, the optimal network architecture for $\mathrm{CNN}$ has to be selected taking into account the balance of computation time and hardware resources and also depending on applications. This paper explores four network architectures: AlexNet, VGG, Residual Network (ResNet) and Wide Residual Network (WideResNet or WRN).
This paper employs multi-task learning (MTL) to improve the accuracy and reduce the computation time in age and gender prediction. The $\mathrm{CNN}$ architectures are modified to introduce MTL. MTL employs one feature extraction both for age prediction task and gender prediction task, while STL employs independent feature extraction for each task. Then, features output from the shared feature extraction are input to independent fullyconnected layers for each task.

\section{Advantages:}

The advantage of face recognition is its convenient image acquisition procedure compared with other biometric recognition such as iris and fingerprint recognition, since special equipment is not required to capture face images.

\section{Limitations:}

Recognition is that face images are dramatically changed by head pose variations, expression changes, aging, etc., resulting in low recognition accuracy.

\section{Deeply Learned Classifiers for Age and Gender Predictions of Unfiltered Faces}

In this paper, a novel end-to-end CNN approach, to achieve robust age group and gender classification of unfiltered real-world faces.

\section{Contributions:}

This paper proposed a model that uses CNN architecture to predict the age group and gender of human's faces from unfiltered real-world environments. Novel CNN approach addresses the age and gender labels as a set of discrete annotations and train the classifiers that predict the human's age group and gender. Designed a quality and robust image preprocessing algorithm that prepare and preprocess the unfiltered images for the $\mathrm{CNN}$ model and this greatly has a very strong impact on the performance accuracy of age and gender classifiers. The figure shows the pipeline of the framework for age group classification

B. Deep Multi-Task Learning:

\begin{tabular}{lllll}
\hline (C) 2022, IJSREM & | www.ijsrem.com & DOI: & Page 7
\end{tabular}


and gender recognition. Demonstrated that pretraining on large-scale datasets allows an effective training of age and gender CNN model which enable the classifiers to generalize on the test images and then avoid overfitting.

\section{Methodology:}

The method proposes a deeply learned classifiers for age group and gender classification of unfiltered real-life face images. The approach as presented below requires image preprocessing (face detection, landmark detection, and face alignment) stage that preprocess and prepare the face images before they are input into the proposed network. Therefore, the solution is divided into three major steps: image preprocessing, features learning, and classification itself. Intelligent age and gender classifiers tackle the classification task under unfiltered real-world settings. Most of those face images are not aligned and non-frontal and also with different degrees of variations in pose, appearance, lighting, and background conditions. Therefore, those in-the-wild face images need first to be detected, then aligned, and, finally, used as input for the classifiers. The CNN architecture used is a novel six-layer network, comprising four convolutional and two fully connected layers. The CNN design is an end-to-end sequential deep learning architecture, including feature extraction and classification phases. The feature extraction phase has four convolutional layers, with the corresponding parameters, including the number of filters, the kernel size of each filter, and the stride.

\section{Advantages:}

This method improves over the best-reported results by $16.6 \%$ (exact accuracy) and 3.2\% (one-off accuracy) for age group classification and also there is an improvement of $3.0 \%$ (exact accuracy) for gender classification. The CNN model is pretrained on large-scale datasets which allows an effective prediction of age and gender.

\section{Limitations:}

Age and gender predictions of unfiltered real-life faces are yet to meet the requirements of commercial and realworld applications despite of the progress computer vision community keeps making with the continuous improvement of the new techniques that improve the state of the art.

\section{Detection of Gender, Age and Emotion of a Human Image using Facial Features}

This paper proposes a Convolutional Neural Network (CNN) based architecture for age \& gender classification.

\section{Contributions:}

- The IMDB-WIKI dataset which is the largest dataset with real age and gender annotations.

- A novel regression formulation is used with deep classification followed by expected value refinement.

- The DEX system, which is the winner of the LAP 2015 challenge on apparent age estimation.

- Age and gender estimation from face images plays an important role in human and computer vision which has many applications in for example, forensics or social media.

\section{Methodology:}

The main motive of this paper is to develop an automatic age and gender estimation method towards human faces which will continue to possess an important role in computer vision and pattern recognition. Apart from age and gender estimation, facial emotion recognition also plays an important role in computer vision. Non-verbal communication methods such as facial expressions, eye movement and gestures are used in many applications of human computer interaction. In order for computer modeling of human's facial features they planned to predict human facial features using deep CNN and observe how intensity changes on a face from low level to high level. Illustration below shows the system architecture of the proposed model. Using the preprocessing algorithm by Viola-Jones they extracted features of the image which are fed as an input to CNN. With a proper user interface, the result of the prediction is revealed. 


\section{Advantages:}

This approach shows better accuracy in both age and gender classification compared to classifier-based methods. In order for computer modeling of human's emotions are predicted using deep CNN and observed how emotional intensity changes on a human's face. A custom CNN model capable of achieving near-state-of-the-art accuracy of $70.47 \%$ on the FER-2013 test set.

\section{Limitations:}

- Illumination is a very important factor in the model's performance. This suggests that the training set may not truthfully represent the distribution of emotion during less brightness on screen.

- One observation was that certain emotion were failing to classify images which were necessary to rely on fine details in the images (e.g., small facial features orcurves).

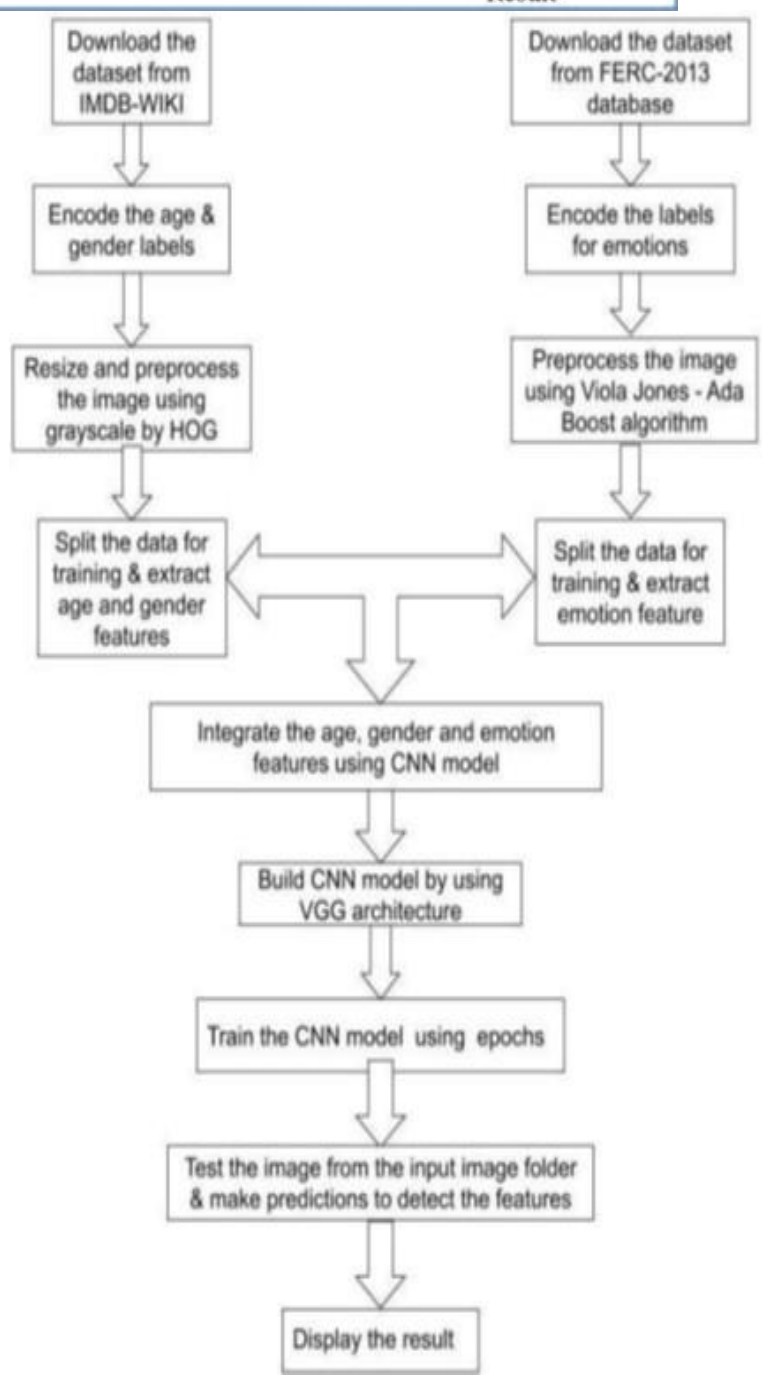




\section{CNN BASED DETECTION OF EMOTION, AGE, GENDER}

This paper proposes a Convolutional Neural Network (CNN) based architecture for emotion, age and gender classification using FER2013 dataset for emotion detection and hdf5 file dataset for age and gender classification.

\section{Contributions:}

This paper suggests that their model can be used for film advice gadget in order to predict the frequency of a variety of age organizations who come to watch films the most and additionally to classify films into distinct genres primarily based on the viewers' emotion. Haar cascade XML file which is a classifier used to become aware of a precise object from the webcam. OpenCV connects to the webcam which consumer can use to scan their faces for classification of age, gender and emotion. This method uses ResNet structure as an

alternative of VGG16 for age or inception v3 for gender classification. ResNet helps in coping with the education error generated as the networks get deeper. In this method ResNet structure is used as an alternative of VGG16 for age or inception $\mathrm{v} 3$ for gender classification, this helps in high rate of accuracy.

\section{Methodology:}

\section{EMOTION DETECTION:}

FER2013 is a Kaggle dataset that incorporates labeled 3589 check images, 28709 educate images. The information augmentation is not required due to the fact the dataset has been constructed with vast vary of images. The database holds grayscale pics of human faces. Switch mastering is not used due to the fact that the dataset consists of grayscale photos and doesn't healthy in three channels pre-trained models. Three convolutional layers are used. Input [48x48x1] includes the pixel values of given image. Hence snap shots have width equal to 48 , peak equal to 48 , and with one colour channel.

\section{AGE AND GENDER CLASSIFICATION:}

An hdf5 file as dataset is used which has 10,000 images. The photographs have been labeled into male, woman and a number of age groups. Here resnet neural community structure is used to instruct this model. ResNet is used to add a giant wide variety of layers with sturdy performance. A community containing residual blocks, every layer is an enter to the subsequent layer and also inputs into the layers about 2-3 hops away. If the wide variety of layers maintains increasing, the accuracy will have a tendency to saturate at sure factor and after a factor it will step by step decrease. This is now not simply due to the fact of over fitting. It indicates that shallow networks are higher at studying than deeper networks. So, the skipconnections skips the coaching of few layers and are additionally referred to as residual connections as proven in above image. By such as omit connections in community architecture, the community is made to pass by education for the layers which are no longer useful and don't add a whole lot cost in common accuracy.

\section{Advantages:}

- The accuracy rate for age and gender classification is obtained about $95 \%$ accuracy rate.

- While the accuracy rate of emotion is bit less than the other's with about $90 \%$ in estimation.

\section{Limitations:}

The database used holds grayscale pictures of human faces. Therefore, it is difficult to identify the details in the human's face.
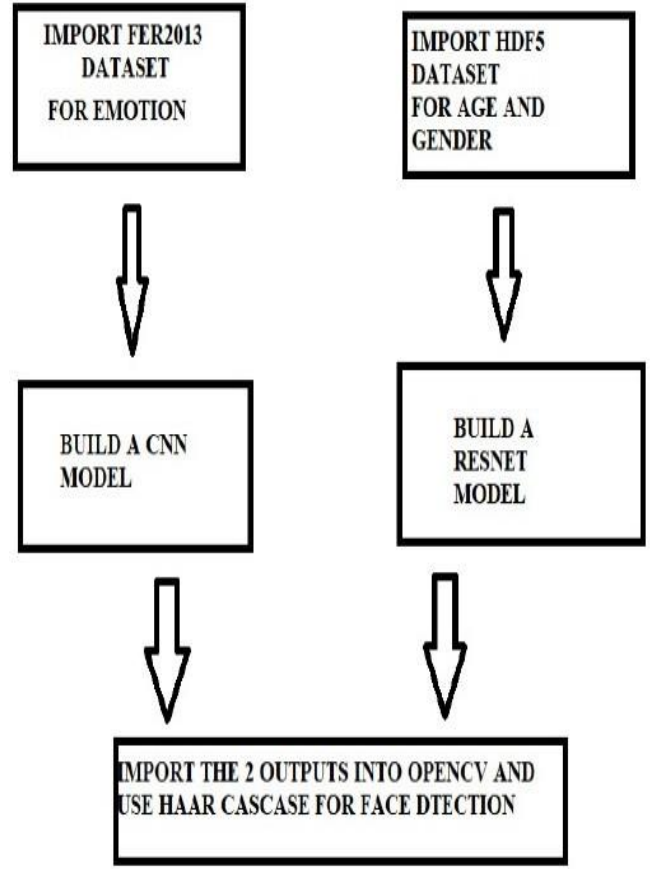


\section{Facial Emotion Recognition Using Deep Learning: \\ Review and Insights}

This paper proposes an application that can be used to help retail industries adjust purchase lists and operation modes to increase sales and improve service quality.

\section{Contributions:}

This system can be applied in retail industries, helping merchants build their own databases to analyze shopping preferences and shopping experiences of users with different ages and genders, and then adjust purchase lists or operating models in a timely manner. Through the data obtained, merchants can categorize customers, and count their shopping preferences, so that to design targeted marketing strategies and in-store activities. This system can provide valuable feedback for in-store promotions by tracking customer emotional responses, enabling retailers to improve product categorization and improve service quality.

\section{Methodology:}

The age and gender of face images were trained using the IMDB-WIKI Database and used a pre-trained VGG network and proposed a novel regression algorithm for the classification of ages. The essence is that after the 101 categories between 0-100, the scores obtained are multiplied by $0-100$, and the final results are summed to obtain the final identified age. Using the IMDB Database, the correct rate for testing gender predictions can reach $96 \%$.

\section{Advantages:}

- The prediction accuracy rate for gender is the highest, which is more than $95 \%$.

- Followed by gender, the accuracy rate can reach more than $86 \%$ in ageestimation.

\section{Limitations:}

The accuracy of emotion estimation is low, not more than $75 \%$. For the prediction of age, it is assumed that the error within plus or minus 2 years is considered to be correct, which gave more accuracy in predicted age

\section{Applications}

Gender, age and emotion recognition can be applied in following use cases:

- The advertisements can be specialized based on the age and the gender of the person on the phone.

- It also can help identify suspects in criminal cases or at least

1. Input Image

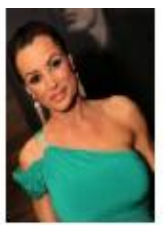

2. Face Detection

3. Cropped Face
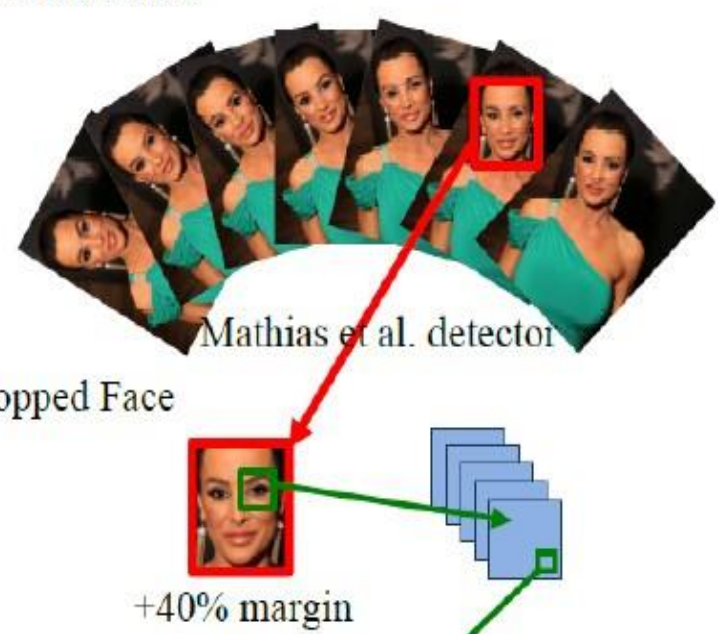

4. Feature Extraction and Prediction

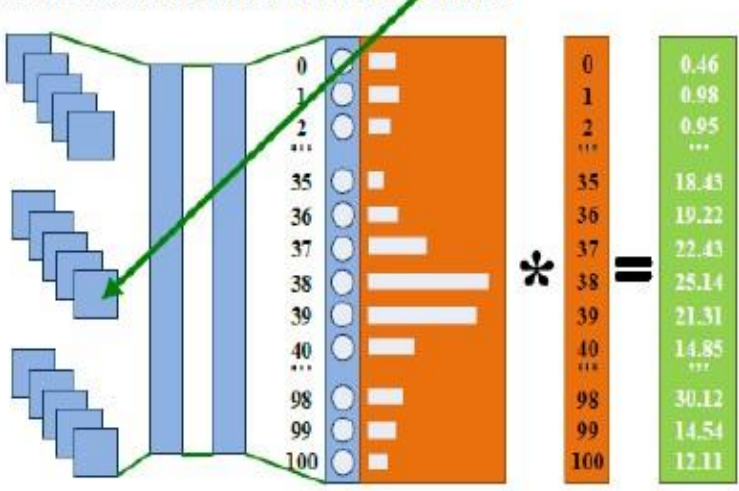

$$
\text { VGG-16 }
$$

architecture

$$
\begin{gathered}
\text { Softmax } \\
\text { expected value }
\end{gathered}
$$

it can minimize the number of suspects.

- It can provide valuable feedback for in-store promotions by tracking customer emotional responses, enabling retailers to improve product categorization and improve service quality.

- Presently, Apple is currently working on providing 
emotional support natively on Apple Products by visually capturing the face via device's onboard camera, processing it and providing necessary help.

\section{Challenges}

- Misclassifications might occur because people from different ethnic groups have different facial characters, which might slightly alter the actual age from the predicted age.

- The use of glasses might affect the emotion classification by interfering with the features learned.

- The database used may holds grayscale pics of human faces.

- Extracting features from one face to another is a difficult and sensitive task in order to have a better classification.

- Overfitting is a trivial problem usually comes when machine learning or deep-learning based methods with limited collection of face images of the dataset.

\section{Conclusion}

The proposed framework is capable of emotion recognition, age estimation and gender recognition simultaneously in a fast and efficient way. As a part of outcome, an android application is developed and a flask application is developed to deploy web application. Android app has the Web-view of the flask application. Misclassifications might occur because people from different ethnic groups have different facial characters, which might slightly alter the actual age from the predicted age. The use of glasses might affect the emotion classification by interfering with the features learned.

Finally, it would be interesting to implement this model on a live business service. This project can be applied in social media to customize advertisements and content, in ecommerce to customize product listing and in entertainment industry to customize OTT (over the top) content.

\section{References}

- M. M. Taghi Zadeh, M. Imani and B. Majidi, "Fast Facial emotion recognition Using Convolutional Neural Networks and Gabor Filters, " 20195 th Conference on Knowledge Based Engineering and Innovation (KBEI), 2019, pp. 577-581, doi: 10.1109/KBEI.2019.8734943.

- $\quad$ P. Smith and C. Chen, "Transfer Learning with Deep CNNs for Gender Recognition and Age Estimation," 2018 IEEE International Conference on Big Data (Big Data), 2018, pp. 2564-2571, doi: 10.1109/BigData.2018.8621891.

- $\quad$ N. Zhou, R. Liang and W. Shi, "A Lightweight Convolutional Neural Network for Real-Time Facial Expression Detection," in IEEE Access, vol. 9, pp. 5573-5584, 2021, doi: 10.1109/ACCESS.2020.3046715.

- $\quad$ E. Eidinger, R. Enbar and T. Hassner, "Age and Gender Estimation of Unfiltered Faces, " in IEEE Transactions on Information Forensics and Security, vol. 9, no. 12, pp. 2170-2179, Dec. 2014, doi: 10.1109/TIFS.2014.2359646.

- V. Carletti, A. Greco, G. Percannella and M. Vento, "Age from Faces in the Deep Learning Revolution," in IEEE Transactions on Pattern Analysis and Machine Intelligence, vol. 42, no. 9, pp. 2113-2132, 1 Sept. 2020, doi: 10.1109/TPAMI.2019.2910522.

- J. Jang, H. Cho, J. Kim, J. Lee and S. Yang, "Facial Attribute Recognition by Recurrent Learning With Visual Fixation," in IEEE Transactions on Cybernetics, vol. 49, no. 2, pp. 616-625, Feb. 2019, doi: 10.1109/TCYB.2017.2782661.

- S. ESCALERA et al., "Guest Editorial: The Computational Face," in IEEE Transactions on Pattern Analysis and Machine Intelligence, vol. 40, no. 11, pp. 2541-2545, 1 Nov. 2018, doi: 10.1109/TPAMI.2018.2869610.

- H. Zhang, A. Jolfaei and M. Alazab, "A Face Emotion Recognition Method Using Convolutional Neural Network and Image Edge Computing, " in IEEE Access, vol. 7, pp. 159081-159089, 2019, doi: 10.1109/ACCESS.2019.2949741.

- P. Chiranjeevi, V. Gopalakrishnan and P. Moogi, "Neutral Face Classification Using Personalized Appearance Models for Fast and Robust Emotion Detection," in IEEE Transactions on Image Processing, vol. 24, no. 9, pp. 2701-2711, Sept. 2015, doi: 10.1109/TIP.2015.2421437.

- E. Şafak and N. Barışçı, "Age and Gender Prediction Using Convolutional Neural Networks," 
2018 2nd International Symposium on Multidisciplinary Studies and Innovative Technologies (ISMSIT), 2018, pp. 1-7, doi:

- A. Atanassov and D. Pilev, "Pre-trained Deep Learning Models for Facial Emotions Recognition," 2020 International Conference Automatics and Informatics (ICAI), 2020, pp. 1-6, doi: 10.1109/ICAI50593.2020.9311334.

- $\quad$ R. Helaly, M. A. Hajjaji, F. M'Sahli and A. Mtibaa, "Deep Convolution Neural Network Implementation for Emotion Recognition System," 2020 20th International Conference on Sciences and Techniques of Automatic Control and Computer Engineering (STA), 2020, pp. 261-265, doi: 10.1109/STA50679.2020.9329302.

- I. Lasri, A. R. Solh and M. E. Belkacemi, "Facial Emotion Recognition of Students using Convolutional Neural Network, " 2019 Third International Conference on Intelligent Computing in Data Sciences (ICDS), 2019, pp. 1-6, doi: 10.1109/ICDS47004.2019.8942386.

- P. Aiswarya, Manish and P. Mangalraj, "Emotion recognition by inclusion of age and gender parameters with a novel hierarchical approach using deep learning, " 2020 Advanced Communication Technologies and Signal Processing (ACTS), 2020, pp. 1-6, doi: 10.1109/ACTS49415.2020.9350479.

- S. Aydın, "Deep Learning Classification of NeuroEmotional Phase Domain Complexity Levels Induced by Affective Video Film Clips, " in IEEE Journal of Biomedical and Health Informatics, vol. 24, no. 6, pp. 1695-1702, June 2020, doi: 10.1109/JBHI.2019.2959843.

- X. Wang, A. Mohd Ali and P. Angelov, "Gender and Age Classification of Human Faces for Automatic Detection of Anomalous Human Behaviour, " 2017 3rd IEEE International Conference on Cybernetics (CYBCONF), 2017, pp. 1-6, doi: 10.1109/CYBConf.2017.7985780.

- $\quad$ https://ieeexplore.ieee.org/search/searchresult.js $\mathrm{p}$ ?newsearch=true\&queryText=gender\%20age $\% 20$ emotion\%20cnn

- $\quad$ https://www.lucidchart.com/pages/examples/flo wchart-maker.

- $\quad$ https://www.kaggle.com/.

https://data.vision.ee.ethz.ch/cvl/rrothe/imdb-wiki/. 